\title{
The National Cholesterol Education Program Adult Treatment Panel III Guidelines
}

\author{
ROBERT J. LIPSY, PharmD, FASHP, BCPS
}

\begin{abstract}
Summary
Coronary heart disease (CHD) persists as a major cause of morbidity and mortality in the United States, with more than $40 \%$ of all deaths each year directly attributed to the disease. Dyslipidemia is recognized as a major risk factor for the development and progression of CHD, with clinical trials clearly demonstrating the public health and economic benefits of favorable cholesterol modification. As a result of this evidence, the National Cholesterol Education Program (NCEP) has developed guidelines for the detection, evaluation, and treatment of high blood cholesterol in adults. The most recent of the NCEP recommendations, the Adult Treatment Panel III (ATP III) guidelines, were released in May 2001 and build on the earlier editions and reiterate the importance of low-density lipoprotein cholesterol (LDL-C) reduction to modify CHD risk. New features of the guidelines include the identification of CHD risk equivalents; lower treatment target goals; an emphasis on conditions conferring a higher risk for CHD, such as the metabolic syndrome; and a scoring system for calculating CHD risk. The ATP III emphasis on risk assessment will result in a substantial increase in the number of patients considered at risk for $\mathrm{CHD}$ and will expand the number eligible for lifestyle and drug intervention.
\end{abstract}

KEYWORDS: Atherosclerosis, Cholesterol, Coronary heart disease, Dyslipidemia, LDL-C

\section{Target Audience}

Managed care pharmacists and other health care practitioners

\section{Learning Objectives}

Upon completion of this program, the participant should be able to 1. review the issues of underidentification and undertreatment of dyslipidemia and discuss how optimal dyslipidemia therapy can improve outcomes and likely reduce comorbidity of related disease,

2. evaluate the impact of the updated NCEP guidelines on clinical practice and discuss the clinical challenges associated with reaching the ATP III goals, 3. describe how cholesterol metabolism is impacted by various lipid pathways, 4. discuss a novel therapeutic approach to lowering cholesterol and explain the rationale for the use of a new class of lipid-lowering agents,

5. review current treatment modalities and patient profiles for dyslipidemia, and 6. describe standard-of-care approaches to improving dyslipidemia treatment outcomes and describe methods for clinical pharmacists to improve therapeutic strategies that enhance overall patient outcomes.

\section{Author Correspondence}

ROBERT J. LIPSY, PharmD, FASHP, BCPS, Manager, Clinical Pharmacy, Health Net of Arizona, 930 North Finance Center Dr., Tucson, Arizona 85710. Tel: (520) 258-7321; Fax: (520) 258-5194; E-Mail: Robert.J.Lipsy@az.health.net

Copyright@ 2003, Academy of Managed Care Pharmacy. All rights reserved.

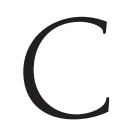
oronary heart disease (CHD) persists as the single leading cause of death among Americans, and the reduction of CHD risk is a critical public health endeavor. In the United States, more than 500,000 Americans succumb to CHDrelated causes each year. ${ }^{1} \mathrm{CHD}$ also places a significant financial burden on the U.S. economy, with the yearly direct and indirect costs of the disease estimated to be in excess of $\$ 111$ billion. Dyslipidemia is recognized as a major modifiable risk factor for the development and progression of CHD. Data from epidemiological studies have indicated that lower cholesterol levels, particularly lower low-density lipoprotein cholesterol (LDL-C) levels, are associated with lower overall risk of $\mathrm{CHD}$ morbidity and mortality ${ }^{2-5}$ Based on this evidence, the National Cholesterol Education Program (NCEP) released the updated Adult Treatment Panel III (ATP III) guidelines in May 2001 to provide pharmacists and other health care professionals with an up-todate approach for the detection, evaluation, and management of dyslipidemia. ${ }^{6}$ Building upon ATP I (1988) and ATP II (1993), ATP III (2001) pays increased attention to the identification and quantification of risk for $\mathrm{CHD}$ and thereby vastly increases the number of Americans eligible for therapies designed to modify lipids and reduce risk. With these changes, ATP III will challenge pharmacists and the health care system to identify at-risk patients, implement effective therapy, and ensure that patients meet target goals.

\section{New Features of the NCEP ATP III Guidelines}

As with previous editions, ATP III provides an evidence-based approach for the detection, evaluation, and management of lipid disorders. ATP III emphasizes LDL-C reduction as the primary target of therapy and advocates that the intensity of lipid modification therapy be adjusted to the degree of risk. ATP III also reiterates the importance of lifestyle changes, such as weight loss, dietary modifications, and increased physical activity, in reducing CHD risk. New features of the guidelines include the use of a Framingham risk assessment tool to evaluate the 10-year probability of experiencing a CHD event; identification of CHD risk equivalents, such as diabetes and peripheral vascular disease; more aggressive lipid target goals; and the recognition that patients with the metabolic syndrome should be provided intensified lipid modification therapy.

One of the hallmarks of ATP III is the categorization of patients into 3 groups based on the overall risk for experiencing a CHD event in 10 years. This approach allows treatment thresholds and lipid target goals to be matched to the patient's degree of risk. Patients with $\mathrm{CHD}$ and $\mathrm{CHD}$ risk equivalents are 
considered at highest risk, those with 2 or more risk factors are considered at moderate risk, and patients with 0 to 1 risk factor are at a low 10-year risk. ATP III also recognized the role played by high-density lipoprotein cholesterol (HDL-C) and triglycerides in modifying CHD risk and raised the target for HDL-C from $<35 \mathrm{mg} / \mathrm{dL}$ to $<40 \mathrm{mg} / \mathrm{dL}$ and lowered the target goal for triglycerides to $200 \mathrm{mg} / \mathrm{dL}$.

\section{CHD Risk Assessment}

ATP III advocates the use of a Framingham risk scoring system to estimate a patient's 10-year risk for a coronary event. Point scores are calculated according to the presence of 6 major CHD risk factors (age, gender, total cholesterol, systolic blood pressure, HDL-C, and smoking status), with each risk factor worth a certain number of points. When added together, the sum yields an estimate of the risk for experiencing a coronary event in 10 years. A properly conducted assessment places patients into one of the 3 risk categories and forms the basis for all subsequent treatment decisions

Patients with documented CHD and CHD risk equivalents are automatically placed in the highest risk category (10-year CHD risk $>20 \%$ ). CHD risk equivalents carry a risk for a major coronary event considered to be equal to that of patients with established CHD. CHD risk equivalents include diabetes, peripheral vascular disease, symptomatic carotid artery disease, and abdominal aortic aneurysm. The LDL-C treatment goal for patients in this high-risk category is $<100 \mathrm{mg} / \mathrm{dL}$.

In patients without documented CHD or CHD equivalents, assessment of $\mathrm{CHD}$ risk is essential to identify therapeutic goals. Patients with 2 or more major risk factors are considered to be at a moderately increased risk for CHD but with a 10-year risk of $10 \%$ to $<20 \%$. Therapy for these patients should be sufficient to enable patients in this category to achieve an LDL-C target of $<130 \mathrm{mg} / \mathrm{dL}$. Patients at the lowest risk are those with one or fewer major risk factors. In all but rare cases, these individuals have a 10 -year risk of $<10 \%$. The target LDL-C level in this group of patients is $<160 \mathrm{mg} / \mathrm{dL}$.

\section{Current Treatment Trends}

With the increased emphasis on risk assessment and intensified new treatment goals, it is estimated that the number of patients eligible for CHD risk reduction through lipid modification therapy in the United States will increase to nearly 65 million. The most effective approach to $\mathrm{CHD}$ risk reduction is one that matches the intensity of therapy to the degree of risk. Two primary modalities advocated by ATP III for lowering LDL-C, and therefore CHD risk, are therapeutic lifestyle changes (TLC) and drug therapy.

\section{Therapeutic Lifestyle Changes}

First-line therapy for most patients without CHD or CHD risk equivalents and $<2$ risk factors is TLC. Eating a healthy diet, participating in regular physical activity, smoking cessation, and

\section{TABLE 1 Overview of the ATP III Guidelines}

- Identifies LDL-C reduction as the primary goal of lipid-modifying therapy

- Evaluates risk of experiencing CHD event in next 10 years

- Targets therapy toward patients at greatest risk, particularly those with established CHD and risk equivalents, including diabetes

- Emphasizes a multifaceted approach to therapy, including therapeutic lifestyle changes (TLC)
Focus on Multiple Risk Factors

- Identifies diabetes without CHD as a cardiovascular risk equivalent

- Uses Framingham projections of 10-year absolute risk to identify certain patients with $>2$ risk factors for more intensive treatment

- Identifies patients with metabolic syndrome as candidates for intensified TLC

Modification of Lipid and Lipoprotein Classification

- Identifies LDL-C $<100 \mathrm{mg} / \mathrm{dL}$ as optimal

- Raises categorically low HDL-C from $<35 \mathrm{mg} / \mathrm{dL}$ to $<40 \mathrm{mg} / \mathrm{dL}$

- Lowers triglyceride cutpoints to give attention to moderate elevations

\section{Support for Implementation}

- Recommends complete lipoprotein profile as preferred initial test (total, LDL-C, HDL-C, triglycerides)

- Encourages use of plant stanols/sterols and soluble fiber as therapeutic dietary options to enhance LDL-C lowering

- Presents strategies for promoting adherence to therapeutic lifestyle changes and drug therapies

- Once LDL-C goal is achieved, recommends treatment beyond LDL-C lowering for patients with triglycerides $\geq 200 \mathrm{mg} / \mathrm{dL}$

weight loss have all been shown to be effective in reducing LDL-C and CHD risk. Dietary changes should include a reduction of saturated fats, reduction of intake of dietary cholesterol to $<200 \mathrm{mg} /$ day, addition of approximately 2 grams of plant sterols/stanols each day, and incorporating approximately $10 \mathrm{~g}$ to $125 \mathrm{~g} /$ day of viscous fiber into the diet. Weight reduction can also reduce LDL-C cholesterol levels and ameliorate the risk factors associated with the metabolic syndrome. Physical activity raises HDL-C levels and decreases the concentration of very low-density lipoprotein cholesterol (VLDL-C) and triglycerides. Smoking cessation can result in an elevation of HDL-C and reduction of CHD risk.

\section{Drug Therapy}

ATP III recognizes the limitations of TLC and encourages the addition of drug therapy if lifestyle modifications fail to achieve goal after 3 months. Most high-risk patients ( $>20 \%$ chance of 
CHD in 10 years) will require concomitant application of drug therapy and TLC from the onset of treatment. For patients at the highest risk for coronary events, the LDL-C threshold for initiation of therapy is $>130 \mathrm{mg} / \mathrm{dL}$ (after a 3-month trial of TLC). Drug therapy is optional for patients with LDL-C between $100 \mathrm{mg}$ and $129 \mathrm{mg} / \mathrm{dL}$, and prescribers are encouraged to use professional clinical judgment in determining the most appropriate approach to risk reduction in these patients. Data from the recent Heart Protection Study have led many experts to recommend early initiation of statins in patients with CHD and CHD risk equivalents, even when the baseline LDL-C is $<130 \mathrm{mg} / \mathrm{dL}$. For patients with moderate risk without $\mathrm{CHD}$ or $\mathrm{CHD}$ risk equivalents, but with $>2$ major risk factors and a 10-year risk of $10 \%$ to $20 \%$, the treatment threshold is $>130 \mathrm{mg} / \mathrm{dL}$. For patients at moderate risk, with a 10 -year risk $<10 \%$, the LDL-C threshold is $>160 \mathrm{mg} / \mathrm{dL}$. For patients without CHD and with 0 to 1 major risk factor, drug treatment should be considered if LDL-C cholesterol is $>190 \mathrm{mg} / \mathrm{dL}$ after 3 months of TLC, with a goal of $<160 \mathrm{mg} / \mathrm{dL}$. In all cases of drug therapy, TLC should continue to be maintained and reinforced.

\section{Current Lipid-Modifying Drugs}

There are currently 3 classes of lipid-modifying drugs recommended by ATP III for the reduction of LDL-C: bile acid sequestrants; nicotinic acids; and 3-hydroxy-3-methylglutaryl coenzyme A reductase inhibitors, or statins. A fourth class, the fibric acid derivatives (fibrates), are not recommended because they have minimal direct effect on lowering LDL-C. However, ATP III does recommend fibrates for patients with very high triglycerides to reduce the risk of acute pancreatitis and for patients with certain other types of dyslipidemia, and fibrates are, therefore, discussed below.

Statins, currently the most widely used lipid-modifying agent, decrease LDL-C by $18 \%$ to $55 \%$ in a dose-dependent manner. These agents exert their effect by inhibiting cholesterol synthesis and promoting LDL-C uptake from the circulation via activation of liver LDL-C receptors. Currently, there are 5 statins on the market. These products are primarily differentiated by the degree of LDL-C lowering elicited by each dose. Several large clinical outcome trials have demonstrated unequivocally that statin use reduces the incidence of CHD events, including myocardial infarction, coronary death, stroke, and total mortal$i^{2}{ }^{2-5}$ These agents are also highly cost effective, especially in high-risk patients. ATP III recommends statins as first-line agents when LDL-lowering drugs are indicated to achieve LDL treatment goals.

Bile acid sequestrants are another commonly used agent that can be used as monotherapy or as add-on therapy to statins. Monotherapy with sequestrants can lead to attainment of treatment goal when moderate reductions in LDL-C are required. However, when more aggressive LDL-C reduction is required, sequestrants must be used in combination with statins. Although generally safe, adherence to a therapeutic regimen containing sequestrants is limited because of poor tolerability and inconvenient dosing.

A third class of agents is nicotinic acid or niacin. Niacin provides a moderate LDL-C-lowering action, but the primary utility of these agents is in combination with statins for patients who have elevated triglycerides and/or low HDL-C. Niacins are also limited in their effectiveness because of compliance issues resulting from poor tolerability. However, the tolerability of niacin therapy can be improved by using either sustained release formulations of the drug or by slowly increasing the dose and providing patients with instructions on how to minimize side effects.

Fibric acids or fibrates are a fourth class of lipid-modifying agents. They are not recommended as primary therapy for LDL-C reduction but are recommended for persons with very high triglycerides to reduce the risk of acute pancreatitis. They also can be recommended for persons with dysbetalipoproteinemia (elevated beta-VLDL). Fibrate therapy should be considered an option for treatment of persons with established CHD who have low levels of LDL-C and atherogenic dyslipidemia. They also should be considered in combination with statin therapy in persons who have elevated LDL-C and atherogenic dyslipidemia. While ATP III does not recommend fibrates in patients who require LDL-C reduction, these agents are useful in patients with combined forms of hyperlipidemia and are especially effective in patients who have severe hypertriglyceridemia. Poor tolerability with fibrates, such as gemfibrozil, can limit adherence to fibrate therapy and therefore reduce the effectiveness of these agents. Limited data suggest that the risk of myopathy may be lowered by substituting the newer fibric acid derivative fenofibrate for gemfibrozil. However, the incidence of myopathy with fenofibrate-statin therapy has not been well studied.

Statins remain the first-line choice of therapy for lipid modification. In most cases, they are safe, effective, and well tolerated, particularly at low doses. However, despite the success of statins in favorably modifying lipids and reducing coronary events, alternative agents are needed for patients unable to tolerate statins. In these cases, prescribers have been forced to use bile acid sequestrants, niacin, or fibrates. However, these alternative agents vary in their effectiveness in reducing LDL-C due to low efficacy of the agent or poor compliance because of undesired side effects.

A promising new alternative therapy has recently received U.S. Food and Drug Administration approval. Phase 2 data suggest that ezetimibe, the first selective inhibitor of intestinal cholesterol absorption, appears to have significant potential for use as monotherapy in patients at low risk for CHD who require only a modest reduction in their LDL-C or for those who do not tolerate statin therapy. ${ }^{7}$ In addition, ezetimibe, when used in combination with a low-dose statin in patients at moderate to high risk for $\mathrm{CHD}$, can elicit a reduction in LDL-C comparable to those seen at the highest statin doses. ${ }^{8}$ Furthermore, ezetim- 
ibe has a safety profile similar to placebo.

Implementation of the ATP III Guidelines

Implementation of the ATP III guidelines will present an enormous challenge to pharmacists and the health care system. Adherence to previous guidelines suggests that achieving the aggressive new detection and treatment goals will be difficult. The ATP II guidelines, although much less complex, were rarely followed in patients with CHD, let alone in a patient with subclinical disease. Data from the Lipid Assessment Treatment Project demonstrated that only $18 \%$ of patients with CHD achieved ATP II goals and that $<40 \%$ of all patients on lipidmodification therapy receive sufficient lipid lowering to reduce CHD risk. ${ }^{9}$ Because overall adherence and goal achievement was low with previous guidelines, the inherent challenge in achieving ATP III goals is clear.

In an attempt to improve cholesterol management, many institutions have developed multidisciplinary lipid clinics with physicians, nurses, pharmacists, and dietitians working together to target, treat, and manage patients at highest risk for CHD. These clinics have been successful in increasing the number of patients achieving NCEP target goals and reducing CHD risk when compared to patients receiving usual care.

\section{Summary and Conclusion}

The NCEP ATP III report updates the clinical guidelines for the detection and treatment of lipid disorders. While the emphasis remains on reducing long-term CHD risk by lowering LDL-C, new features of the guidelines include a scoring system for calculating CHD risk as well as the identification of CHD risk equivalents. The ATP III emphasis on risk assessment will substantially increase the number of individuals considered to be at risk for CHD and will expand the number who will be eligible for lifestyle and drug intervention. Pharmacists have the opportunity to impact patient care by positively encouraging cholesterol evaluation screening and treatment upon patient admission to the hospital and in the clinic. In addition, pharmacists can assist patients through education on the need for risk-factor interventions and achieving target goals and by encouraging adherence and persistence to therapy.

\section{DISCLOSURES}

Dr. Lipsy received an honorarium for participating in the symposium on which this article is based. He disclosed having no financial interest/relationships with commercial entities related to his presentation materials.

\section{REFERENCES}

1. American Heart Association. 2001 Heart and Stroke Statistical Update. Dallas, Texas: American Heart Association; 2001:11.

2. Scandinavian Simvastatin Survival Study Group. Randomised trial of cholesterol lowering in 4,444 patients with coronary heart disease: the Scandinavian Simvastatin Survival Study (4S). Lancet. 1994;344:1383-89.

3. West of Scotland Coronary Prevention Study Group. Influence of pravastatin and plasma levels on clinical events in the West of Scotland Coronary Prevention Study (WOSCOPS). Circulation. 1998;97:1440-45.

4. Downs, JR, Clearfield M, Weis S, et al. Primary prevention of acute coronary events with lovastatin in men and women with average cholesterol levels: results of AFCAPS/TexCAPS. Air Force/Texas Coronary Atherosclerosis Prevention Study. JAMA. 1998;279(20):1615-22.

5. Sacks FM, Maye LA, Davis BR, et al. Relationship between plasma LDL concentrations during treatment with pravastatin and recurrent coronary events in the Cholesterol and Recurrent Events trial. Circulation.1998; 97:1446-52.

6. Expert Panel on the Detection, Evaluation, and Treatment of High Blood Cholesterol in Adults. Executive summary of the third report of the National Cholesterol Education Program (NCEP) Expert Panel on Detection, Evaluation, and Treatment of High Blood Cholesterol in Adults. JAMA. 2001; 285:2486-97

7. Bays H, Drehobl M, Rosenblatt S, et al. Low-density lipoprotein cholesterol reduction by SCH 58235 (ezetimibe), a novel inhibitor of cholesterol absorption, in 234 hypercholesterolemic subjects: results of a dose-response study [abstract]. Atherosclerosis. 2000;151:133.

8. Davis HR Jr, Watkins RW, Comptons DS, et al. The cholesterol absorption inhibitor ezetimibe (SCH 58235) and lovastatin synergistically lower plasma cholesterol and inhibit the development of atherosclerosis [abstract]. J Am Coll Card. 2000;35(2 suppl):252

9. Pearson TA, Laurora I, Chu H, Kafonek S. The lipid treatment assessment project (L-TAP): a multicenter survey to evaluate the percentages of dyslipidemic patients receiving lipid-lowering therapy and achieving low-density lipoprotein cholesterol goals. Arch Int Med. 2000;160(4):459-67. 